EPJ Web of Conferences 37, 01005 (2012)

DOI: $10.1051 /$ epjconf/20123701005

(C) Owned by the authors, published by EDP Sciences, 2012

\title{
Status and hadron physics program of J-PARC
}

\author{
K. Ozawa ${ }^{\mathrm{a}}$
}

High Energy Research Organization (KEK), Japan

\begin{abstract}
Current status and hadron physics programs of Japan Accelerator Research Complex (J-PARC) are reported. Several physics programs are proposed at the Hadron Hall of J-PARC. Strangeness and hyper nuclear physics is undertaken at K1.8 beam line. Study of meson nucleon bound system is underway at K1.8BR beam line. After the earthquake, all beam line components and experimental setups are reassembled. Protons are successfully accelerated in the last December and hadron physics experiments are resumed in this February. In this manuscript, status of on-going experiment and near future plans of such physics programs are reported. Especially, a new beam line for a primary protons and high momentum secondary particles is proposed to study meson properties in nucleus.
\end{abstract}

\section{Introduction}

Many experiments related to hadron physics are being performed and proposed at the Hadron Hall of Japan Accelerator Research Complex (J-PARC). The summary of proposed experiments can be found here [1]. There are several beam lines at the hall as shown in Fig. 1.

The primary protons are delivered to the production target which is placed at the front of the hall and produce several secondary particles. Secondary particles is used as a beam for each experiment. At the left side of the hall, there are two beam lines, such as K1.8 and K1.8BR. The main purpose of these beam lines is kaon beam experiments and the beam lines have a good $\mathrm{K} / \pi$ separation ability.

${ }^{a}$ e-mail: ozawa@post.kek.jp

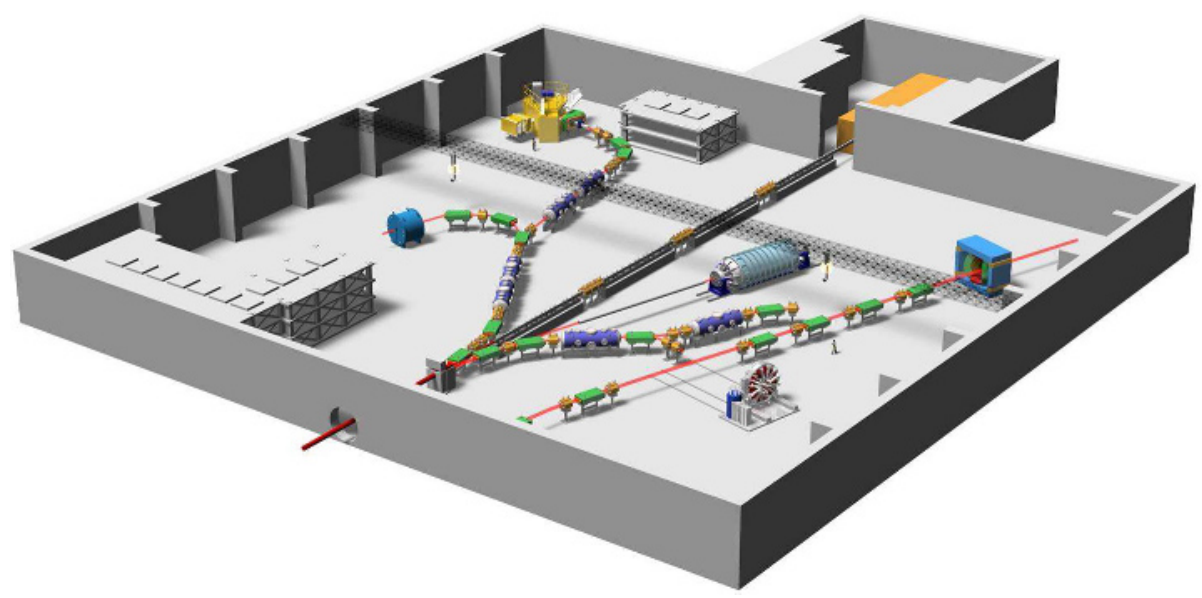

Fig. 1. Schematic view of the Hadron Hall. 
Table 1. Characteristics of beam lines at the Hadron Hall of J-PARC.

\begin{tabular}{lcll}
\hline $\begin{array}{l}\text { Beam } \\
\text { Line }\end{array}$ & $\begin{array}{l}\text { Maximum } \\
\text { Momentum }[\mathrm{GeV} / c]\end{array}$ & $\begin{array}{l}\text { Particle } \\
\text { Species }\end{array}$ & $\begin{array}{l}\text { Physics } \\
\text { Topics }\end{array}$ \\
\hline K1.8 & 2.0 & $\mathrm{~K}, \pi, \mathrm{p}, \ldots$ & Hyper Nucleus \\
$\mathrm{K} 1.8 \mathrm{BR}$ & 1.2 & $\mathrm{~K}, \pi, \mathrm{p}, \ldots$ & Kaon bound system \\
K1.1 & 1.1 & $\mathrm{~K}, \pi, \mathrm{p}, \ldots$ & Hyper Nucleus \\
$\mathrm{K} 1.1 \mathrm{BR}$ & 0.8 & $\mathrm{~K}, \pi, \mathrm{p}, \ldots$ & T-violations \\
KL & & Neutral Kaon & $\mathrm{K}_{L}$ rare decays. \\
High-p & 30 & primary proton & Meson mass in nucleus \\
& 15 & un-separated (mainly,$\pi)$ & Heavy baryon \\
\hline
\end{tabular}

At the left side of the hall, there are two charged particle beam lines and one neutral kaon beam line (KL). Charged particle beam lines, called K1.1 and K1.1BR, have relatively short length and have great benefits for low momentum kaon beam to suppress in-flight decays of kaon.

In addition to these beam lines for secondary particles, one beam line for primary protons is being prepared. Small fraction of primary protons are separated at upstream of the main beam line and delivered to the hadron hall for experimental use directly. When an additional thin production target is placed at the separation point, the beam line can be used as a high momentum secondary beam line.

Each beam line has specific beam characteristics and physics programs. Characteristics of beam line are shown in Tab. 1. In the following sections, several physics programs using these beam line are described. KL and K1.1BR beam lines are mainly for particle physics experiments and detailed explanation for these beam lines are omitted in this manuscript.

After the earthquake, all beam line components and experimental setups are reassembled. Protons are successfully accelerated in the last December and hadron physics experiments are resumed in this February.

\section{Pentaquark}

The first hadron experiment at J-PARC is searching for a pentaquark. In 2003, the LEPS collaboration observed a narrow resonance at $1540 \mathrm{MeV} / c^{2}$ in the $K^{-}$missing mass spectrum for the $\gamma n \rightarrow K^{+} K^{-} n$ reaction on a carbon target [2]. This new particle named as pentaquark, $\Theta^{+}$, because the resonance have very narrow width and it can be interrupted as five quarks states, $u u d d \bar{s}$. Since then, many positive and negative experimental results are reported. The existence of $\Theta^{+}$is an urgent problem in the field of hadron physics.

The J-PARC E19 experiment aims to search for the pentaquark $\Theta^{+}$in $\pi p \rightarrow K^{-} \mathrm{X}$ reaction [3]. The first physics run is performed from October to November 2010 at the K1.8 beam line and pion beam. The beam momentum of the pion is set to be $1.92 \mathrm{GeV} / c$ and analyzed by a beam line spectrometer. Typical intensity of the beam is $\sim 10^{6}$ pions/pulse with a duty factor of $16 \%$. Total $7.8 \times 10^{10}$ pions are irradiated to the liquid hydrogen target, whose interaction length is $0.86 \mathrm{~g} / \mathrm{cm}^{2}$. Scattered kaons are detected by the Superconducting Kaon Spectrometer (SKS) which provides the momentum resolution of $0.2 \%$ (FWHM) with a wide acceptance of $100 \mathrm{msr}$. Performances of the spectrometer are evaluated with $\Sigma^{+}$productions. The measured resolution of missing mass in $\pi^{+} p \rightarrow \mathrm{K}^{+} \mathrm{X}$ reaction is $1.9 \mathrm{MeV}$ (FWHM). The missing mass resolution of $\Theta$ measurements is evaluated as $1.4 \mathrm{MeV}$ (FWHM).

Results of the experiment are already published in [4]. No peak structure is observed in obtained spectra. The data can be described with a simulated background from known hadron productions and a three-body phase space. After the acceptance correction, obtained data is fitted with a Gaussian peak and a polynomial background and an upper limit of the production cross section is evaluated. The obtained upper limit is $0.26 \mu \mathrm{b} / \mathrm{sr}$ at $90 \%$ confidence level in the mass region of $1.51-1.55 \mathrm{GeV} / \mathrm{c}^{2}$. 


\section{Kaon Bound States}

Interactions between $\bar{K}$ and nuclei attract wide interests in recent years. Especially, structures of the $\Lambda(1405)$ are still under discussions. The resonance has been interpreted as a quasi-bound state, embedded in $\Sigma-\pi$ continuum originally. According to a recent coupled channel approach based on the ansatz that a $K^{-} p$ bound state is located at $1405 \mathrm{MeV} / c^{2}$, strong attractive $\bar{K} N$ interactions are deduced and a strongly bound $\bar{K}$ nuclear states are predicted [5]. While, theories based on chiral dynamics claim that the $K^{-} p$ quasi-bound state locates at $1420 \mathrm{MeV}$ or higher [6].

To investigate $\bar{K} N$ interactions and the structure of $\Lambda(1405)$, current experimental data are not sufficient [7]. New experimental data for elementary $\bar{K}$ induced reactions are essentially important. At the K1.8BR beam line of J-PARC hadron hall, three new experiments are being performed.

The J-PARC E15 experiment searches for a simple kaonic bound state, $K^{-} p p$ [8]. In the experiment, the bound state will be formed in $K^{-}+\mathrm{He}^{3} \rightarrow K^{-} p p+n$ reactions. The experiment performs an exclusive measurement, such as a missing mass spectroscopy using emitted neutrons and invariant mass spectroscopy via an expected decay $K^{-} p p \rightarrow \Lambda p \rightarrow p \pi^{-} p$. To identify the bound state, resolutions of the missing mass and the invariant mass measurements are designed to be less than 10 $\mathrm{MeV} / c^{2}(\sigma)$ and $20 \mathrm{MeV} / c^{2}(\sigma)$, respectively.

An isospin dependence of $K^{-}$nucleus strong interaction at a low energy limit is studied by the JPARC E17 experiment [9]. The experiment measures $3 \mathrm{~d} \rightarrow 2 \mathrm{p} \mathrm{X}$-rays from $K^{-3} \mathrm{He}$ and $K^{-4} \mathrm{He}$ atoms using high-resolution silicon drift X-ray detectors (SDDs) with the world's highest precision and study a shift of the X-rays induced by strong interaction. Required energy resolution of X ray measurements is $185 \mathrm{eV}$ (FWHM) at $6.4 \mathrm{keV}$ which is already achieved in the previous experiment KEK-PS E570 [10].

In addition to above two experiments, the J-PARC E31 experiment is approved to measure a spectral function of the $\Lambda(1405)$ produced in a $\bar{K} N \rightarrow \pi \Sigma$ channel via in-flight $\left(K^{-}, n\right)$ reaction on a deuteron target [11].

A dedicated spectrometer is designed and constructed at the $\mathrm{K} 1.8 \mathrm{BR}$ beam line to satisfy all above experimental requirements. The spectrometer consists of a high precision beam line spectrometer, a liquid helium target system, a Cylindrical Detector System (CDS) that is placed around the target to detect decay particles, and a neutron time-of-flight counter array located $\sim 15 \mathrm{~m}$ away from the target position. Pilot runs of the experiment are already performed in this summer. As results of pilot runs, a clear peak of $\Lambda \rightarrow p \pi^{-}$decay is identified and the mass resolution of $3.3 \mathrm{MeV} P(\sigma)$ for $\Lambda$ is obtained. Obtained resolutions satisfy experimental requirements.

\section{Mesons in Nucleus}

Meson properties in nucleus give a fruitful information in hadron physics. For example, $\eta \mathrm{N}$ system is suggested to have a strong coupling to the $\mathrm{N}^{*}$ resonance in the threshold region [12] and nature of $\eta$ mesic nucleus reflects in-medium properties $N^{*}$ properties, which is strongly related to the chiral symmetry of baryons [13]. New experiment is being proposed at J-PARC [14].

Another good example is measurements of $\omega$ meson in nucleus. An experiment to study properties of $\omega$ meson in nucleus is proposed [15] and approved as the J-PARC E26 experiment. The experiment searches a nuclear $\omega$ bound states in $\mathrm{A}\left(\pi^{-}, \mathrm{n}\right) \omega \mathrm{X}$ reaction and missing mass measurements using emitted forward neutrons. A strongly bounded state are predicted by several theories [16]. When a binding energy of a $\omega$ bound state in nucleus is measured, it can be interpreted to an optical potential and gives a phenomenological information about interactions between $\omega$ meson and nuclei.

The proposed experiment also measure invariant mass spectra of $\omega$ meson in nucleus with $\omega \rightarrow \pi^{0} \gamma$ decay mode in low momentum region. Using missing mass information in forward neutron measurements, the generation process of $\omega$ meson can be identified. In addition, $\omega$ meson is generated in recoil less kinematics and the momentum of generated $\omega$ meson is very limited within the Fermi motion. Also, if $\omega$ meson is bounded in nucleus, which can be observed using the forward neutron measurement, the kinematic condition of $\omega$ meson in nucleus is established very clearly. 


\section{Vector Meson Mass in Nucleus}

The origin of the hadron mass has been drawing strong interests in nuclear and particle physics and its naively understood as a spontaneous breaking of the chiral symmetry. In hot and/or dense matter, this broken symmetry will be restored either partially or completely and, hence, properties of hadrons, such as mass, decay modes and life time, can be modified. Therefore, we can study the origin of hadron mass and chiral properties of QCD medium by measuring in-medium properties of mesons. Especially, mass spectra of vector mesons is directly connected to anti-quark quark condensates [17], which is an order parameter of chiral symmetry. Relatively large mass modification is theoretically expected even at nuclear density [18].

Among several experimental results, the KEK-PS E325 experiment observes a modification of $\phi$ mesons in nuclear medium on an invariant mass spectrum of $e+e-$ pairs in $12 \mathrm{GeV} p+A$ interactions [19]. The observed modification can be interpreted as the mass of $\phi$ meson decreased by $3.4 \%$ and the width of $\phi$ meson broden by 3.6 times at the normal nuclear density. Still, statistics of obtained spectra is not enough and kinematical dependence of the modification can not be discussed. Thus, a new experiment to achieve 100 times larger statistics is being prepared at J-PARC, as the J-PARC E16 experiment [20].

The J-PARC E16 experiment measures invariant mass spectra of $e^{+} e-$ pairs in $30 \mathrm{GeV} p+A$ reactions. The experiment uses a high momentum beam line which is being constructed in a few years at the Hadron Hall. The beam line can provide a $30 \mathrm{GeV}$ primary protons with a typical intensity of $10^{10}$ protons per spill ( 6 seconds). The beam intensity is 10 times higher than KEK experiment and Gas Electron Multiplier (GEM) technology [21] is fully adopted to cope with such high intensity beam experiment. A particle trajectory is measured with the GEM tracker and required mass resolution is $5 \mathrm{MeV} c^{2}$, which is improved by factor of 2 from the KEK experiment. An electron identification is performed with the Hadron Blind Detector (HBD), which has the same configuration as the PHENIX experiment developed [22].

Expected spectra with mass modification of $\phi$ meson is shown in Fig. 2. To calculated the spectra, the same mass modifications as KEK results are assumed. Improved mass resolution helps to see a clear double peak. In addition, a proton target is used to figure out experimental e ffects precisely.

As results of huge statistics and small ambiguity in measurements, clear mass spectra of $\phi$ meson in nucleus can be provided as a basic information for hadron properties in nucleus.
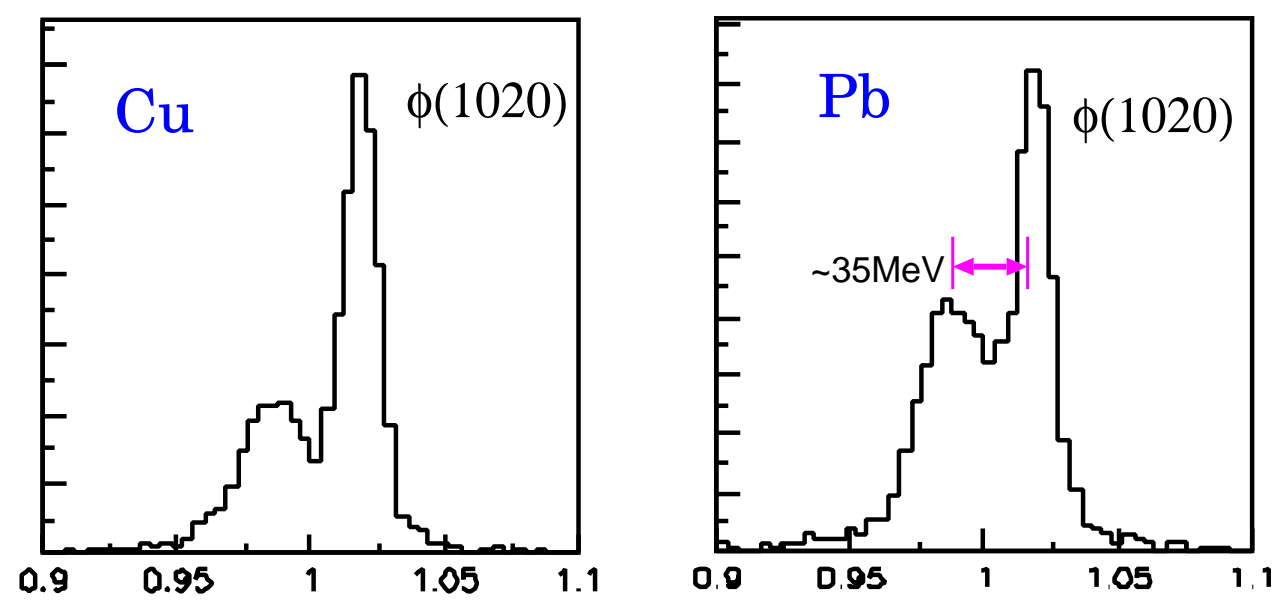

Fig. 2. Expected mass spectra for $\mathrm{Cu}$ and $\mathrm{Pb}$ targets in the E16 experiment. 


\section{Summary}

There are several interesting physics programs at J-PARC Hadron Facility. The pentaquark search experiment using $\pi p \rightarrow K^{-} \mathrm{X}$ reaction and no corresponding structure has bee observed on a missing mass spectrum. The search for the kaonic nuclei will be performed at the K1.8BR beam line and the property of $K^{-}$pp system is studied both in the invariant and missing mass spectra. The preliminary results show that detector performance is good enough for the experiment. In addition, several experiments for meson bound states are prepared and planned. In addition, new beam line for primary protons is being constructed and study of meson mass in nucleus will be done.

As a conclusion, there are great opportunities to study important problems in the field of hadron physics at J-PARC.

\section{References}

1. http://j-parc.jp/researcher/Hadron/en/PAC_for_NuclPart_e.html

2. T. Nakano et al., Phys. Rev. Lett. 91, (2003) 012002

3. J-PARC E19 proposal, http://j-parc.jp/researcher/Hadron/en/pac 0606/pdf/p19-Naruki.pdf

4. K. Shirotori et al., Phys. Rev. Lett. to be published.

5. Y. Akaishi et al., Phys. Rev. C 65, (2002) 044005

N.V. Shevchenko et al., Phys. Rev. C 76, (2007) 044004

6. D. Jido et al., Nucl. Phys. A 725, (2003) 181

V.K. Megas et al., Phys. Rev. C 74, (2006) 025206

A. Dote et al., Phys. Rev. C 79, (2008) 014003

7. O. Braun et al., Nucl. Phys. B 129, (1977) 1

M. Niiyama et al., Phys. Rev. C 78, (2008) 035202

T. Yamazaki et al., Phys. Rev. Lett. 104, (2010) 132502

8. J-PARC E15 proposal, http://j-parc.jp/researcher/Hadron/en/pac_0606/pdf/p15-Iwasaki.pdf

9. J-PARC E17 proposal, http://j-parc.jp/researcher/Hadron/en/pac_0606/pdf/p17-Hayano.pdf

10. S. Okada et al., Phys. Lett. B 653, (2007) 387

11. J-PARC E31 proposal, http://j-parc.jp/researcher/Hadron/en/pac_0907/pdf/Noumi.pdf

12. W. Grein et al., Nucl. Phys. A 338, (1980) 332

13. D. jido et al., Nucl. Phys. A 671, (2000) 471

14. K. Itahashi et al., LOI, http://j-parc.jp/researcher/Hadron/en/pac_0707/pdf/LoI-itahashi.pdf

15. J-PARC E26 proposal, http://j-parc.jp/researcher/Hadron/en/pac_1001/pdf/KEK_J-PARC-PAC2009-16.pdf

16. E. Marco and W. Weise, Phys. Lett B 502 (2001) 59 H. Nagahiro, D. Jido, and S. Hirenzaki, Nucl. Phys. A 761 (2005) 92

17. T. Hatsuda and S.H. Lee, Phys. Rev. C 46 (1992) R34

18. T. Hatsuda and T. Kunihiro, Phys. Rep. 247 (1994) 221

G.E. Brown and M. Rho, Phys. Rep. 269 (1996) 333

W. Cassing and E.L. Bratkovskaya, Phys. Rep. 308 (1999) 65

19. R. Muto et al., Phys. Rev. Lett. 98, (2007) 042501

20. J-PARC E16 proposal, http://j-parc.jp/researcher/Hadron/en/pac_0606/pdf/p16-Yokkaichi_2.pdf

21. F. Sauli, Nucl. Instr. and Meth. A386, (1997) 531

22. A. Kozlov et al., Nucl. Instr. and Meth. A636, (2011) 99 